\title{
DISCOVERY OF WATER VAPOR IN THE HIGH-REDSHIFT QUASAR APM 08279+5255 AT $z=3.91$
}

\author{
D. C. Lis ${ }^{1}$, D. A. Neufeld ${ }^{2}$, T. G. Phillips ${ }^{1}$, M. Gerin ${ }^{3}$, AND R. Neri ${ }^{4}$ \\ ${ }^{1}$ California Institute of Technology, Cahill Center for Astronomy and Astrophysics 301-17, Pasadena, CA 91125, USA; dcl@caltech.edu, tgp@submm.caltech.edu \\ ${ }^{2}$ Department of Physics and Astronomy, The Johns Hopkins University, 3400 North Charles Street, Baltimore, MD 21218, USA; neufeld@jhu.edu \\ ${ }^{3}$ LERMA, UMR 8112 du CNRS, Observatoire de Paris and Ecole Normale Superiéure, 24 Rue Lhomond, 75231 Paris cedex 05, France; maryvonne.gerin @ lra.ens.fr \\ ${ }^{4}$ IRAM-Institut de Radio Astronomie Millimétrique, 300 rue de la Piscine, 38406 Saint-Martin d'Hères, France; neri@iram.fr, cox@iram.fr \\ Received 2011 June 1; accepted 2011 June 23; published 2011 August 9
}

\begin{abstract}
We report a detection of the excited $2_{20}-2_{11}$ rotational transition of para- $\mathrm{H}_{2} \mathrm{O}$ in APM $08279+5255$ using the IRAM Plateau de Bure Interferometer. At $z=3.91$, this is the highest-redshift detection of interstellar water to date. From large velocity gradient modeling, we conclude that this transition is predominantly radiatively pumped and on its own does not provide a good estimate of the water abundance. However, additional water transitions are predicted to be detectable in this source, which would lead to an improved excitation model. We also present a sensitive upper limit for the hydrogen fluoride (HF) $J=1-0$ absorption toward APM $08279+5255$. While the face-on geometry of this source is not favorable for absorption studies, the lack of HF absorption is still puzzling and may be indicative of a lower fluorine abundance at $z=3.91$ compared with the Galactic interstellar medium.
\end{abstract}

Key words: cosmology: observations - galaxies: active - galaxies: high-redshift - galaxies: individual (APM 08279+5255) - ISM: molecules

\section{INTRODUCTION}

Molecules such as $\mathrm{CO}$ or HCN have been commonly used as tracers of molecular gas in high-redshift galaxies. However, recent observations with the Herschel Space Observatory (Pilbratt et al. 2010) have shown strong spectroscopic signatures from other light hydrides, such as water, $\mathrm{H}_{2} \mathrm{O}^{+}$, or hydrogen fluoride (HF), in nearby active galaxies (e.g., van der Werf et al. 2010). These lines are blocked by the Earth's atmosphere, but can be observed, redshifted, in distant galaxies using the current millimeter and submillimeter facilities. For example, Omont et al. (2011) have recently reported a detection of water in J090302-014127B (SDP.17b) at $z=2.30$.

One of the exciting recent results from HIFI (de Graauw et al. 2010) is the detection of widespread absorption in the fundamental $J=1-0$ rotational transition of HF toward Galactic sources (Neufeld et al. 2010; Phillips et al. 2010; Sonnentrucker et al. 2010; Monje et al. 2011). Fluorine is the only atom that reacts exothermically with $\mathrm{H}_{2}$ (Neufeld et al. 2005; Neufeld \& Wolfire 2009). The product of this reaction, HF, is thus easily formed in regions where $\mathrm{H}_{2}$ is present and its very strong chemical bond makes this molecule relatively insensitive to UV photodissociation. As a result, HF is the main reservoir of fluorine in the interstellar medium (ISM), with a fractional abundance of $\sim 3.5 \times 10^{-8}$ relative to $\mathrm{H}_{2}$ typically measured in diffuse molecular clouds within the Galaxy (Neufeld et al. 2010; Sonnentrucker et al. 2010; Monje et al. 2011).

Interstellar HF was first detected by Neufeld et al. (1997) with the Infrared Space Observatory (ISO). The $J=2-1$ rotational transition was observed in absorption toward Sagittarius B2, at a low spectral resolution using the Long-Wavelength Spectrometer (LWS). The HIFI instrument allows for the first time observations of the fundamental rotational transition of $\mathrm{HF}$ at $1.232476 \mathrm{THz}$ to be carried out, at high spectral resolution. Given the very large Einstein $A$ coefficient $\left(2.423 \times 10^{-2} \mathrm{~s}^{-1}\right.$; critical density $\sim 3 \times 10^{9} \mathrm{~cm}^{-3}$ ), this transition is generally observed in absorption against dust continuum background. Only extremely dense regions with strong IR radiation field could possibly generate enough collisional or radiative excitation to yield an HF feature with a positive frequency-integrated flux. ${ }^{5}$ The HIFI observations corroborate the theoretical prediction that $\mathrm{HF}$ will be the dominant reservoir of interstellar fluorine under a wide range of interstellar conditions. The HF $J=1-0$ transition promises to be an excellent probe of the kinematics of, and depletion within, absorbing material along the line of sight toward bright continuum sources, and one that is uncomplicated by the collisionally excited line emission that is usually present in the spectra of other gas tracers. As suggested by Neufeld et al. (2005), redshifted HF $J=1-0$ absorption may thus prove to be an excellent tracer of the ISM in the high-redshift universe, although only the gas reservoir in front of a bright continuum background can be studied by means of the HF absorption spectroscopy.

Water is another interstellar molecule of key importance in astrophysical environments, being strongly depleted on dust grains in cold gas, but abundant in warm regions influenced by energetic process associated with star formation (see van Dishoeck et al. 2011 and references therein). The excited $2_{20}-2_{11}$ transition of $\mathrm{p}-\mathrm{H}_{2} \mathrm{O}$, with a lower level energy of $137 \mathrm{~K}$, has a frequency of $1.228788 \mathrm{THz}$ and can be observed simultaneously with the $J=1-0$ transition of HF in highredshift systems. Consequently, we have searched for the HF $J=1-0$ and $\mathrm{H}_{2} \mathrm{O} 2_{20}-2_{11}$ transitions, redshifted down to $251 \mathrm{GHz}$, in APM 082791+5255 using the IRAM Plateau de Bure Interferometer (PdBI).

The broad absorption line (BAL) quasar APM 082791+5255 at $z=3.9118$, with a true bolometric luminosity of $(0.7-3) \times$ $10^{14} L_{\odot}$, is one of the most luminous objects in the universe (Downes et al. 1999). CO lines up to $J=11-10$ have been detected using the IRAM $30 \mathrm{~m}$ telescope. IRAM PdBI high spatial resolution observations of the CO $J=4-3$ and 9-8 lines, and of the $1.4 \mathrm{~mm}$ dust continuum have been presented by Weiß et al. (2007). The line fluxes in the CO ladder and the dust continuum fluxes are fit well by a two-component model

\footnotetext{
5 HF emission has recently been reported in the extreme environment of the nearby ultraluminous galaxy Markarian 231 in Herschel/SPIRE observations at low spectral resolution (van der Werf et al. 2010).
} 
that invokes a cold component at $65 \mathrm{~K}$ with a high density of $n\left(\mathrm{H}_{2}\right)=1 \times 10^{5} \mathrm{~cm}^{-3}$, and a warm, $\sim 220 \mathrm{~K}$, component with a density of $1 \times 10^{4} \mathrm{~cm}^{-3}$. Weiß et al. argue that the molecular lines and the dust continuum emission arise from a very compact ( $r \simeq 100-300 \mathrm{pc}$ ), highly gravitationally magnified $(m \simeq 60-110)$ region surrounding the central active galactic nucleus (AGN). Part of the difference relative to other high- $z$ quasi-stellar objects may therefore be due to the configuration of the gravitational lens, which gives us a high-magnification zoom right into the central $200 \mathrm{pc}$ radius of APM $08279+5255$ where IR pumping plays a significant role for the excitation of the molecular lines.

High angular resolution (0.'3) Very Large Array observations of the CO $J=1-0$ emission in APM 08297+5255 (Riechers et al. 2009) reveal that the molecular emission originates in two compact peaks separated by $\lesssim 00^{\prime} 4$ and is virtually co-spatial with the optical/near-infrared continuum emission of the central AGN. This morphological similarity again indicates that the molecular gas is located in a compact region, close to the AGN. Riechers et al. (2009) present a revised gravitational lens model of APM $08297+5255$, which indicates a magnification by only a factor of four, in contrast to much higher magnification factors of $\sim 100$ suggested in earlier studies. Their model suggests that the $\mathrm{CO}$ emission originates from a $\sim 550 \mathrm{pc}$ radius circumnuclear disk viewed at an inclination angle of $\lesssim 25^{\circ}$, or nearly face-on. The total molecular mass is then $1.3 \times 10^{11} M_{\odot}$.

Weiß et al. (2007) first pointed out the importance of infrared pumping for the excitation of HCN in APM 08279+5255. Subsequent observations of Riechers et al. (2010) reveal surprisingly strong $J=6-5$ emission of $\mathrm{HCN}, \mathrm{HNC}$, and $\mathrm{HCO}^{+}$in the host galaxy, providing additional evidence that these transitions are not collisionally excited. Riechers et al. (2010) argue that the high rotational lines of HCN can be explained by infrared pumping at moderate opacities in a $\sim 220 \mathrm{~K}$ warm gas and dust component. These findings are consistent with the overall picture in which the bulk of the gas and dust is situated in a compact, nuclear starburst, where both the AGN and star formation contribute to the heating.

Prior to the observations reported here, water had not been detected in APM 08279+5255. However, Wagg et al. (2006) give an upper limit of $0.7 \mathrm{Jy} \mathrm{km} \mathrm{s}^{-1}(3 \sigma)$ for the ground state $1_{10}-1_{01}$ ortho- $\mathrm{H}_{2} \mathrm{O}$ line.

\section{OBSERVATIONS}

Observations of APM 08279+5255 presented here were carried out on 2010 June 22, September 21-22, and December 15 , using the PdBI. Visibilities were obtained in the CD set of configurations of the six-element array in June and December and with a four-element subarray in September, totaling $4.9 \mathrm{hr}$ of on-source observations.

Data reduction and calibration were carried out using the GILDAS software package in the standard antenna-based mode. The passband calibration was measured on 3C454.3, and amplitude and phase calibration were made on $0749+540$, $0836+716$, and $0917+449$. The absolute flux calibration, performed using MWC349 as the primary calibrator (2.55 Jy at $250 \mathrm{GHz}$ ), is accurate to within $10 \%$. Point-source sensitivities of $4.5 \mathrm{mJy} \mathrm{beam}^{-1}$ were obtained in channels of $20 \mathrm{MHz}$, consistent with the measured system temperatures (200-300 K). The conversion factor from flux density to brightness temperature in the $1^{\prime \prime} .6 \times 1^{\prime \prime} .3\left(\right.$ P.A. $=22^{\circ}$ ) synthesized beam is $9.1 \mathrm{~K}\left(\mathrm{Jy} \mathrm{beam}^{-1}\right)^{-1}$.

\section{RESULTS}

Figure 1 (upper panel) shows a spectrum of APM $08279+5255$ near the rest-frame frequency of the HF $J=1-0$ transition, integrated over the PdBI image, which also covers frequencies of the para- $\mathrm{H}_{2} \mathrm{O} \quad 2_{20}-2_{11}$ and $\mathrm{HCN} J=14-13$ transitions, in addition to HF. The continuum is detected with a high signal-to-noise ratio. The integrated flux density, computed from emission free channels, is $34 \pm 0.55 \mathrm{mJy}$, consistent with the previous measurements of the source spectral energy distribution (Weiß et al. 2007). No HF absorption is seen, with a $3 \sigma$ upper limit of $1.5 \mathrm{Jy} \mathrm{km} \mathrm{s}^{-1}$, assuming a full width at half-maximum (FWHM) line width of $500 \mathrm{~km} \mathrm{~s}^{-1}$, as implied by earlier $\mathrm{CO}$ observations.

The integrated line and continuum fluxes given above impose a $3 \sigma$ upper limit of 0.092 for the velocity-averaged HF $J=$ 1-0 optical depth (velocity-integrated optical depth $\tau d v=$ $46 \mathrm{~km} \mathrm{~s}^{-1}$ ). The corresponding column density of cold HF in front of the continuum source can then be computed using Equation (3) of Neufeld et al. (2010) to be $N(\mathrm{HF})<1.1 \times$ $10^{14} \mathrm{~cm}^{-2}$. Given the typical Galactic $\mathrm{HF} / \mathrm{H}_{2}$ abundance ratio of $\sim 3.5 \times 10^{-8}$, this value would imply an average $\mathrm{H}_{2}$ column density $\sim 3 \times 10^{21} \mathrm{~cm}^{-2}$ lying in front of the continuum source in APM $08279+5255$. This value is three orders of magnitude below the beam-averaged $\mathrm{H}_{2}$ column density inferred from the dust continuum flux observed toward the source. ${ }^{6}$

The para- $\mathrm{H}_{2} \mathrm{O} \quad 2_{20}-2_{11}$ line is clearly detected with the integrated line flux density of $6.7 \pm 0.8 \mathrm{Jy} \mathrm{km} \mathrm{s}^{-1}$. A Gaussian fit gives a line width of $510 \pm 70 \mathrm{~km} \mathrm{~s}^{-1}$, consistent with that of CO. Figure 1 (lower panel) shows the spatial distribution of the para- $\mathrm{H}_{2} \mathrm{O} 2_{20}-2_{11}$ emission (red contours) superposed on a gray-scale image of the dust continuum. The line and continuum emission peak toward sources A and B of Riechers et al. (2009). The small offset between the $\mathrm{H}_{2} \mathrm{O}$ and continuum emission is not significant at the spatial resolution of the present observations. The implication for water excitation in APM $08279+5255$ is discussed below.

Some excess emission above the continuum level is seen near the frequency of the HCN $J=14-13$ line; however, the result does not constitute a detection at the sensitivity limit of the present observations.

\section{WATER EXCITATION}

In modeling the water line flux observed from APM $08279+5255$, we have computed the $\mathrm{CO}$ and $\mathrm{H}_{2} \mathrm{O}$ line luminosities expected for an isothermal, constant density medium. We solved the equations of statistical equilibrium for the $\mathrm{H}_{2} \mathrm{O}$ and $\mathrm{CO}$ level populations, making the large velocity gradient (LVG) approximation and treating the effects of radiative trapping with an escape probability method. We adopted the rate coefficients of Yang et al. (2010) and Faure \& Josselin (2008), respectively, for the excitation of $\mathrm{CO}$ and $\mathrm{H}_{2} \mathrm{O}$ in collisions with $\mathrm{H}_{2}$, and we assumed an ortho-to-para ratio (OPR) of 3 for both $\mathrm{H}_{2}$ and $\mathrm{H}_{2} \mathrm{O}$. Following Weiß et al. (2007), we neglect any effects of dust extinction upon the emergent $\mathrm{CO}$ and $\mathrm{H}_{2} \mathrm{O}$ line fluxes; although the dust optical depths at $\mathrm{THz}$ frequencies raise the possibility that such effects could be significant, their importance depends strongly on the geometry of the source and

\footnotetext{
6 In their two-component model for the dust continuum radiation, Weiß et al (2007) derived estimates of $\sim 2.8 \times 10^{9} \mathrm{~m}^{-1} M_{\odot}$ and $680 \mathrm{pc}$, respectively, for the dust mass and magnified radius for APM $08279+5255$, where $m$ is the lens magnification. These values imply an average $\mathrm{H}_{2}$ column density of $1.2 \times 10^{25} \mathrm{~cm}^{-2}$ for an assumed dust-to-gas mass ratio of 100 .
} 

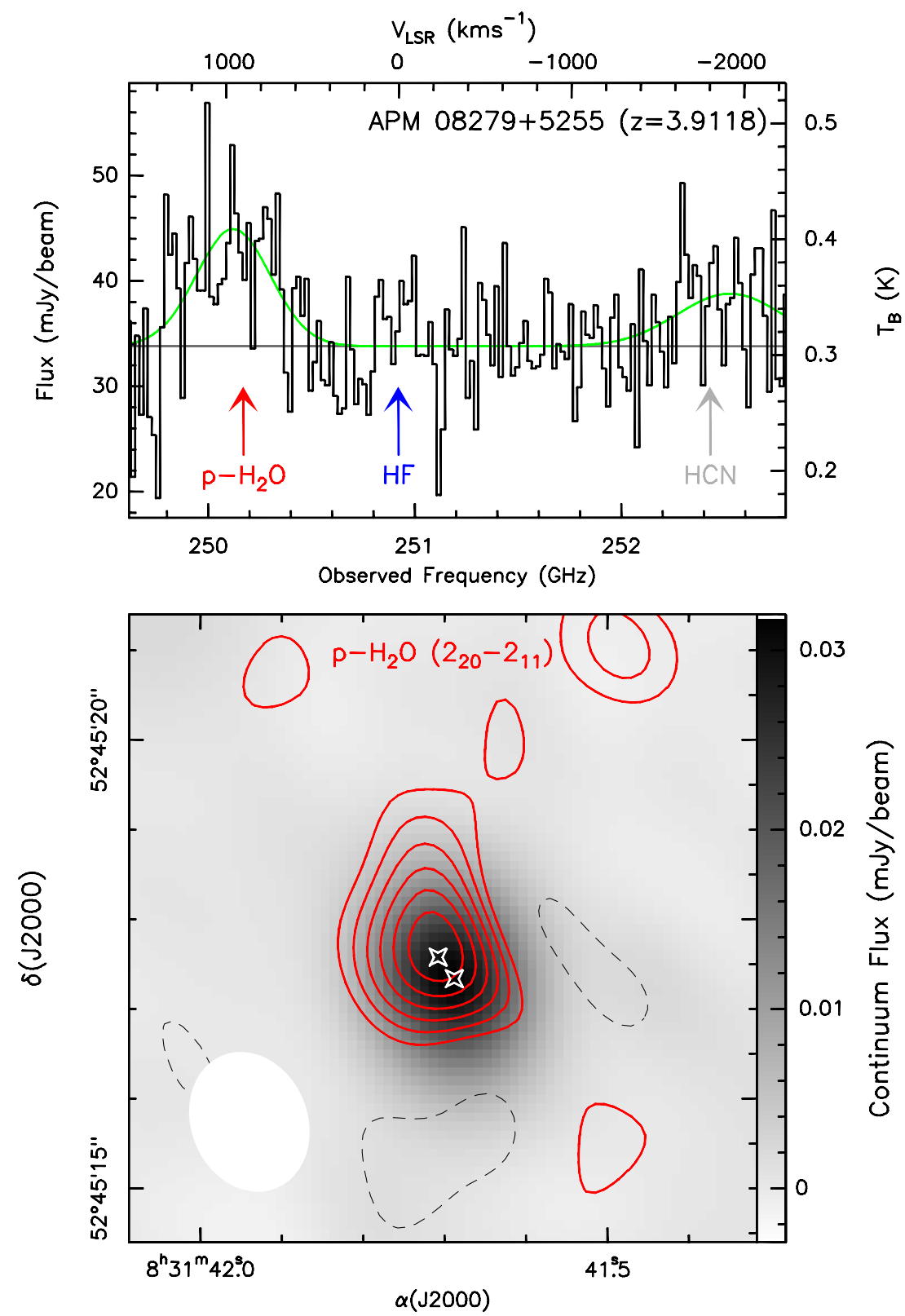

Figure 1. Top: integrated spectrum of APM $08279+5255$. Vertical arrows mark redshifted frequencies of para- $\mathrm{H}_{2} \mathrm{O} 2_{20}-2_{11}$, HF $J=1-0$, and $\mathrm{HCN} J=14-13$, assuming $z=3.9118$. The velocity scale is with respect to the HF $J=1-0$ frequency. HF and HCN lines are not detected. Bottom: distribution of the velocity-integrated para- $\mathrm{H}_{2} \mathrm{O} 22_{20}-2_{11}$ line intensity in APM $08279+5255$ (red contours) superposed on a gray-scale image of the $1.23 \mathrm{THz}$ (rest frame) dust continuum emission. Contour levels are $-2,2,3,4,5,6,7$ times the rms of $0.8 \mathrm{Jy} \mathrm{km} \mathrm{s}^{-1}$ beam $^{-1}$. White symbols mark the locations of sources A and B (Riechers et al. 2009). Synthesized beam is shown as a white ellipse in the lower left corner.

the spatial relationship between the warm dust and the molecular emission region. In Galactic hot cores with $\mathrm{H}_{2}$ column densities comparable to that in APM $08279+5255$, the para$\mathrm{H}_{2} \mathrm{O} 22_{20}-2_{11}$ line can be seen with net-emission flux (Orion KL, NGC6334I), or in absorption (Sagittarius B2), depending on the specific source geometry. A mixture of such regions may contribute to the observed spectrum of APM $08279+5525$, leading to partial cancellation of the emergent line flux. Using the relative strengths of the multiple $\mathrm{CO}$ transitions observed by Weiß et al. (2007) to constrain the gas temperature, density, and velocity gradient, we thereby obtained as bestfit parameters the values $T=10^{2.35} \mathrm{~K}, n\left(\mathrm{H}_{2}\right)=10^{4.2} \mathrm{~cm}^{-3}$, and $d v / d z=1.4 \times 10^{5} n(\mathrm{CO}) / n\left(\mathrm{H}_{2}\right) \mathrm{km} \mathrm{s}^{-1} \mathrm{pc}^{-1}$, respectively. These parameters are very close to those obtained previously by
Weiß et al. (2007) in their single component model for the CO emission detected from this source.

Adopting the same parameters for the water emitting region, we have computed the para- $\mathrm{H}_{2} \mathrm{O} \quad 2_{20}-2_{11} / \mathrm{CO} J=11-10$ line flux ratio as a function of the assumed $n\left(\mathrm{H}_{2} \mathrm{O}\right) / n(\mathrm{CO})$ abundance ratio. In the case of $\mathrm{H}_{2} \mathrm{O}$, the pumping of rotational transitions by far-infrared continuum radiation can strongly affect the predicted line fluxes. The importance of radiative pumping in this source has been discussed previously by Riechers et al. (2010) for the case of HCN, although, in that case, pumping takes place through a low-lying vibrational band. Pumping through pure rotational transitions is relatively much more important for an asymmetric top molecule like water, because such molecules possess a more complex energy level 

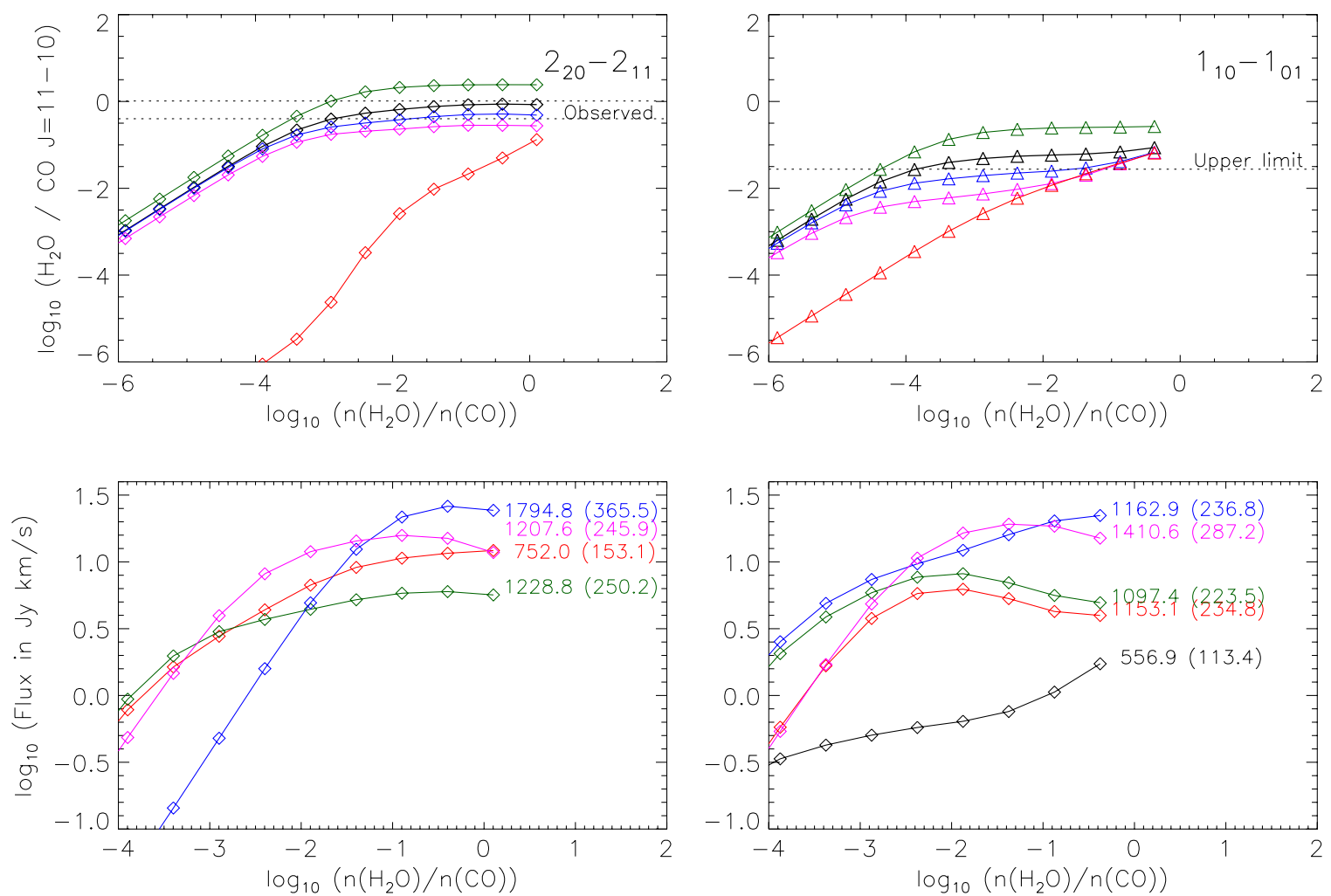

Figure 2. Molecular line ratios predicted by LVG models of APM 08279+5255, as described in the text. Labels in the lower two panels give the rest frequencies in $\mathrm{GHz}$ (redshifted frequencies in parentheses) of water transitions that are predicted to be strong and potentially detectable.

structure than the simple ladder shown by spinless linear or diatomic molecules (such as $\mathrm{HCN}$ and $\mathrm{CO}$ ); furthermore, the lowest vibrational band of water lies at a considerably shorter wavelength $(\sim 6.3 \mu \mathrm{m})$ than that of $\mathrm{HCN}(\sim 14.7 \mu \mathrm{m})$, where the continuum radiation is considerably weaker. The dominance of radiative pumping in rotational transitions of water vapor was also discussed by González-Alfonso et al. (2010), in their recent analysis of the water line emission observed by Herschel toward the starburst galaxy Mrk 231. Under conditions where radiative pumping is dominant, the water line fluxes are almost independent of the gas temperature and density.

Following Riechers et al. (2010), we define $\operatorname{IR}_{\mathrm{ff}}$ as the sky covering factor of the infrared continuum source at the location of the molecular emission region; the mean intensity is then given by modified blackbody of the form $\operatorname{IR}_{\mathrm{ff}}(1-$ $\left.e^{-\tau}\right) B_{v}(220 \mathrm{~K})$, where $B_{v}(T)$ is the Planck function and $\tau=$ $1.05(\mathrm{v} / 1 \mathrm{THz})^{2}$. In Figure 2 (upper left panel), we present the predicted para- $\mathrm{H}_{2} \mathrm{O} 2_{20}-2_{11} / \mathrm{CO} J=11-10$ line flux ratio, as a function of $n\left(\mathrm{H}_{2} \mathrm{O}\right) / n(\mathrm{CO})$ and for several different values of $\mathrm{IR}_{\mathrm{ff}}$ : 0 (red), 0.1 (magenta), 0.25 (blue), 0.5 (black), and 1.0 (green). The results shown in Figure 2 clearly indicate the dominant role of radiative pumping; a detailed analysis indicates the importance of the $1_{11} \rightarrow 2_{20}$ (rest frame $2.97 \mathrm{THz}$ ) transition in directly pumping the $2_{20}$ state of para-water, along with the $2_{02}-3_{31}$ (rest frame $6.45 \mathrm{THz}$ ) transition, which pumps the $3_{31}$ state; the latter can decay subsequently to $2_{20}$. Dotted horizontal lines indicate the para- $\mathrm{H}_{2} \mathrm{O} 2_{20}-2_{11} / \mathrm{CO} J=11-10$ ratio measured in APM08279+5255 and its uncertainty.

The upper right panel of Figure 2 shows entirely analogous results for the $1_{10}-1_{01}$ transition of ortho-water, with the horizontal dotted line indicating the $3 \sigma$ upper limit obtained by Wagg et al. (2006). A comparison of the results shown in the upper panels of Figure 2 indicates that a limited range of parameters is permitted by the measured value of the $2_{20}-2_{11}$ line flux and the upper limit on $1_{10}-1_{01}$. For the $\mathrm{H}_{2} \mathrm{O}$ OPR of 3 assumed here, acceptable fits are obtained for $\mathrm{IR}_{\mathrm{ff}} \sim 0.25$ and $n\left(\mathrm{H}_{2} \mathrm{O}\right) / n(\mathrm{CO})$ in the range $\sim 0.003-0.03$, although the range of acceptable parameters would obviously broaden if OPR values smaller than 3 were permitted. In the regime of interest, the expected $2_{20}-2_{11}$ line fluxes depend only weakly upon the $\mathrm{H}_{2} \mathrm{O}$ abundance, the most important pumping transitions being optically-thick; thus, the exact range of acceptable values for $n\left(\mathrm{H}_{2} \mathrm{O}\right) / n(\mathrm{CO})$ depends strongly upon our estimate of the likely error in the measured line flux. Nevertheless, the $n\left(\mathrm{H}_{2} \mathrm{O}\right) / n(\mathrm{CO})$ ratio inferred for an assumed OPR of 3 is apparently smaller than that typically measured $(\sim 0.1-1)$ in hot core regions within our Galaxy (e.g., Boonman \& van Dishoeck 2003). Observations of additional transitions will be needed to constrain the water OPR and abundance better. The lower panels of Figure 2 present results for several other transitions that are potentially detectable from ground-based observatories, some of which are more strongly dependent upon the water abundance. The results shown in these panels were all obtained for $\mathrm{IR}_{\mathrm{ff}}=0.25$ and $\mathrm{OPR}=3$, and the labels indicate the rest frequencies and-in parentheses-redshifted frequencies in GHz. Our results for transitions of para- and ortho-water appear, respectively, in the left and right panels.

We note that our LVG solution for the $\mathrm{H}_{2} \mathrm{O}$ emission in APM $08279+5525$ not only matches that of Weiß et al. (2007), using independent data, but the deduced velocity gradient $d v / d z$ for the $\mathrm{H}_{2} \mathrm{O}$ emitting region is close to the expected virial value, as defined, for example, by Equation (5) of Greve et al. (2009), $K_{\text {vir }} \sim 4$ (i.e., the dense gas emitting in the $\mathrm{H}_{2} \mathrm{O}$ line emission is near virial equilibrium). 


\section{DISCUSSION}

The absence of detectable HF $J=1-0$ absorption in APM $08279+5255$ is unexpected, given the low column density of $\mathrm{HF}$ required to produce measurable absorption. An important caveat in this analysis is the assumption of a Galactic $\mathrm{HF} / \mathrm{H}_{2}$ ratio that, in turn, is related to the elemental abundance of fluorine. Fluorine nucleosynthesis - and thus the evolution of the fluorine abundance in cosmic time-remains poorly understood, with production in asymptotic giant branch stars (e.g., Cristallo et al. 2009), in Wolf-Rayet stars (Meynet \& Arnould 2000) and neutrino-induced nucleosynthesis in Type II supernovae (Woosley \& Haxton 1998) all proposed as possible mechanisms. While the face-on geometry of APM $08279+5255$ is not favorable for absorption studies, the lack of HF absorption is still puzzling and may be indicative of a lower fluorine abundance in this source compared with the Galactic ISM. Nevertheless, $\mathrm{HF}$ absorption may still prove to be a good tracer of $\mathrm{H}_{2}$ in highredshift sources and additional observations of objects with different geometries, over a wide range of redshifts, are urgently needed.

Our LVG models indicate that the para- $\mathrm{H}_{2} \mathrm{O} 2_{20}-2_{11}$ transition in APM $8279+5255$ is predominantly radiatively pumped. Omont et al. (2011) has reached similar conclusions regarding water excitation in J090302-014127B (SDP.17b) at $z=2.30$. The para- $\mathrm{H}_{2} \mathrm{O} 2_{20}-2_{11}$ line intensity in APM $8279+5525$ is sensitive to the details of the excitation model. Consequently, observations of this single transition do not provide a good estimate of the water abundance. However, our LVG models suggest that many additional water lines should be detectable with the current millimeter-wave facilities. The transitions that are expected to be the strongest (see Figure 2) are $3_{21}-3_{12}$ (rest-frame frequency $1162.2 \mathrm{GHz}), 4_{22}-4_{13}(1207.6 \mathrm{GHz})$, $2_{11}-2_{02}(752.0 \mathrm{GHz})$, as well as two very high energy transitions $6_{24}-6_{15}(1794.8 \mathrm{GHz})$ and $5_{23}-5_{14}(1410.6 \mathrm{GHz})$, which are sensitive to the gas density. With multi-line observations, the excitation conditions and the water abundance will be much better constrained. This excitation scenario can further be tested with observations of the $2.97 \mathrm{THz}$ pumping transition, which is expected to appear in absorption.

This work is based on observations carried out with the IRAM Plateau de Bure interferometer. IRAM is supported by INSU/CNRS (France), MPG (Germany), and IGN (Spain). This research has been supported by NASA through an award issued by JPL/Caltech and by the NSF grant AST-0540882 to the Caltech Submillimeter Observatory. We thank Pierre Cox for allocating Director's Discretionary Time to allow these observations to be carried out and an anonymous referee for constructive and helpful comments.

\section{REFERENCES}

Boonman, A. M. S., \& van Dishoeck, E. F. 2003, A\&A, 403, 1003

Cristallo, S., Straniero, O., Gallino, R., et al. 2009, ApJ, 696, 797

de Graauw, Th., Helmich, F. P., Phillips, T. G., et al. 2010, A\&A, 518, L6

Downes, D., Neri, R., Wiklind, T., Wilner, D. J., \& Shaver, P. A. 1999, ApJ, $513, \mathrm{~L} 1$

Faure, A., \& Josselin, E. 2008, A\&A, 492, 257

González-Alfonso, E., Fischer, J., Isaak, K., et al. 2010, A\&A, 518, L43

Greve, T. R., Papadopoulos, P. P., Gao, Y., \& Radford, S. J. E. 2009, ApJ, 692, 1432

Meynet, G., \& Arnould, M. 2000, A\&A, 355, 176

Monje, R., Emprechtinger, M., Phillips, T. G., et al. 2011, ApJ, 734, L23

Neufeld, D. A., Sonnetrucker, P., Phillips, T. G., et al. 2010, A\&A, 518, L1018

Neufeld, D. A., \& Wolfire, M. G. 2009, ApJ, 706, 1594

Neufeld, D. A., Wolfire, M. G., \& Schilke, P. 2005, ApJ, 628, 260

Neufeld, D. A., Zmuidzinas, J., Schilke, P., \& Phillips, T. G. 1997, ApJ, 488, L141

Omont, A., Neri, R., Lupu, R., et al. 2011, A\&A, 530, L3

Phillips, T. G., Bergin, E. A., Lis, D. C., et al. 2010, A\&A, 518, L109

Pilbratt, G. L., Riedinger, J. R., Passvogel, T., et al. 2010, A\&A, 518, L1

Riechers, D. A., Walter, F., Carilli, C. L., \& Lewis, G. 2009, ApJ, 690, 463

Riechers, D. A., Weiß, A., Walter, F., \& Wagg, J. 2010, ApJ, 725, 1032

Sonnentrucker, P., Neufeld, D. A., Phillips, T. G., et al. 2010, A\&A, 521, L12

van der Werf, P. P., Isaak, K. G., Meijerink, R., et al. 2010, A\&A, 518, L42

van Dishoeck, E. F., Kristensen, L. E., Benz, A. O., et al. 2011, PASP, 123, 138

Wagg, J., Willner, D. J., Neri, R., et al. 2006, ApJ, 651, 46

Weiß, A., Downes, D., Neri, R., et al. 2007, A\&A, 467, 955

Woosley, S. E., \& Haxton, W. C. 1998, Nature, 334, 45

Yang, B., Stancil, P. C., Balakrishnan, N., \& Forrey, R. C. 2010, ApJ, 718, 1062 